\title{
The possibility of resistant hypertension during the treatment of hypertensive patients
}

\author{
Tetsuo Nishikawa, Masao Omura, Jun Saito and Yoko Matsuzawa
}

Patients with poorly controlled hypertension despite taking at least three different kinds of anti-hypertensive drugs, including diuretics, are considered to have resistant hypertension (RH). The prevalence of RH was reported to be $13 \%$ in the Japanese J-HOME study. The incidences of RH in younger and older Japanese individuals should be prospectively investigated in the near future. RH is associated with poor outcomes and various cardiovascular events. In addition, it is frequently associated with older age, obesity, sleep apnea, long-term hypertension, diabetes, dyslipidemia, reduced renal function, microalbuminuria and left ventricular hypertrophy. Some cases of RH exhibit high levels of aldosterone and cortisol, suggesting that endocrine hypertension should be ruled out among RH patients. Carotid baroreceptor activation and renal sympathetic denervation have recently been developed as treatments for $\mathrm{RH}$. In conclusion, we should consider the possibility of RH during the treatment of hypertensive patients who do not achieve appropriate blood pressure control, in order to avoid the early onset of fatal cardiovascular events and reduce medical costs.

Hypertension Research (2013) 36, 924-929; doi:10.1038/hr.2013.107; published online 12 September 2013

Keywords: cardiovascular events; obesity; primary aldosteronism; sleep apnea syndrome; white coat syndrome

\section{INTRODUCTION}

Patients with resistant hypertension $(\mathrm{RH})$ are at risk of poor outcomes. Adherence to medication schedules and treatment intensification improve blood pressure (BP) control; however, little is known about their effects on $\mathrm{RH}$ or their associations with outcomes in this patient group. A retrospective cohort study of two integrated health plans conducted between 2002 and 2006 reported that 1 in 50 patients with incidental hypertension developed $\mathrm{RH} .{ }^{1}$ In addition, the patients who developed $\mathrm{RH}$ were found to be at increased risk of cardiovascular events; thus, greater effort should be made to improve hypertension outcomes in this population. ${ }^{1}$ The misdiagnosis of $\mathrm{RH}$ during hypertension treatment, for example, it being diagnosed as white coat hypertension or non-adherence to anti-hypertensive medication, sometimes occurs in primary care. Thus, we should refocus on what RH is and how it should be treated. Below, we review the recent findings regarding the pathogenesis and treatment of $\mathrm{RH}$.

\section{AVOIDANCE OF HASTY DIAGNOSIS OF RH}

$\mathrm{RH}$ is sometimes diagnosed in patients with spurious resistant HT whose BP was inaccurately measured and/or evaluated. The protocol for measuring BP should always be strictly adhered to. It is essential to confirm whether or not the cuff size is appropriate, the patient is in a proper position, the arm is at a proper height and the patient has had sufficient rest prior to the measurement. Pseudohypertension, a BP reading that erroneously appears highly elevated, is incorrectly diagnosed as $\mathrm{RH}$, especially in elderly patients, because progressive arteriosclerosis, common among the elderly, results in an increase in arterial stiffness, which makes it difficult to compress the arteries without great pressure during the measurement of BP. Thus, in order to make an accurate diagnosis of true $\mathrm{RH}$, it is necessary to carefully consider all of the clinical information about each patient, including the physical characteristics and laboratory findings, as well as the methods used to measure BP.

It is well known that $\mathrm{RH}$ is defined as resistance to treatment when administered three or more different kinds of anti-hypertensive medications, including diuretics, to decrease BP. There is a white coat effect that could result in an overestimation of actual BP during treatment. It is generally accepted that the long-term prognosis of hypertensive patients showing a white coat effect may not be as severe, and with fewer critical cardiovascular complications, as that of patients who have true RH. Ambulatory BP monitoring (ABPM) is useful for evaluating diurnal changes of BP, such as normal or elevated asleep mean. We can differentiate white coat hypertension from persistent hypertension by ABPM, as described in detail below. We should measure BP by using ABPM for an accurate diagnosis of true RH.

It is also well known that uncontrolled hypertension, which is sometimes misdiagnosed as $\mathrm{RH}$, exists in such cases showing nonadherence to lifestyle modification and anti-hypertensive therapy. Furthermore, behavioral medicine has a crucial role in the hypertension management of patients with spurious resistant HT; before a diagnosis of $\mathrm{RH}$ is made, individual patient education and 
empowerment, and non-adherence to medications and lifestyle modification should be improved. Another problem is 'patient-physician adherence'. We need to focus on the various cognitive and behavioral factors or characteristics of each hypertensive patient that may have an influence on communication and treatment. We should always consider the possibility of spurious resistant HT among the patients with persistent and uncontrolled hypertension before making a definitive diagnosis of RH. A correct diagnosis of true $\mathrm{RH}$ can only be made after ensuring that $\mathrm{BP}$ was accurately measured, proper adherence to the patient-physician relationship and administration of anti-hypertensive medications, and differentiating white coat hypertension from severe persistent hypertension among patients by $A B P M$.

\section{DEFINITION AND PREVALENCE}

We often encounter patients who display persistently high BP values despite taking anti-hypertensive drugs. Hypertensive patients whose BP does not return to normal after lifestyle modification and treatment with at least three different kinds of anti-hypertensive drugs, including diuretics, are diagnosed with $\mathrm{RH}$ and/or refractory hypertension. Comparative studies have suggested that uncontrolled $\mathrm{RH}$ should be referred to as refractory hypertension, and controlled $\mathrm{RH}$ should be termed resistant or difficult-to-control hypertension, ${ }^{2}$ although RH is essentially considered to be the same condition as refractory hypertension. The prevalence of $\mathrm{RH}$ was reported to be $13 \%$ in the Japanese J-HOME study and ranged from $30-50 \%$ in the following Western studies: ALLHAT, ${ }^{3}$ CONVINCE, ${ }^{4}$ LIFE, ${ }^{5}$ INSIGHT ${ }^{6}$ and VALUE. ${ }^{7}$ We need to establish a definitive definition of $\mathrm{RH}$ that is based on the findings of home BP studies and/or measurements obtained with $\mathrm{ABPM}$ and to clarify the physiological relevance and causative mechanisms of poor BP control under anti-hypertensive drug administration. Recent analyses of the data obtained during the National Health and Nutrition Examination Survey (NHANES), which involved subjects drawn from representative samples of the adult, non-institutionalized, civilian population, estimated the prevalence of $\mathrm{RH}$ to be $8.9 \pm 0.6 \%$ of the US hypertensive population in 2003-2008. ${ }^{8}$ A time-sequence comparison of the NHANES data from 1998 through 2008 suggested that, unlike hypertension, RH is becoming more prevalent (for example, it displayed an incidence of $20.7 \%$ from 2005 to 2008) due to the aging of the general population and the increasing frequency of obesity. ${ }^{8}$

\section{PATHOGENESIS OF RH}

We sometimes encounter RH patients who are complicated with obesity, sleep apnea syndrome, primary aldosteronism (PA) or white coat syndrome as well as $\mathrm{RH}$ patients who have been prescribed inappropriate anti-hypertensive drugs or are not following the prescribed treatment schedule. In such cases, we ask the patients to modify their lifestyles, for example, to adopt a low-salt diet or exercise more, and attempt to improve their adherence to the prescribed treatment schedule. It is essential to refer such patients to specialist hypertension clinics, as $\mathrm{RH}$ patients are often at high risk of cardiovascular events and/or secondary hypertension.

$\mathrm{RH}$ can occur for the following reasons: (1) inadequate measurement of BP due to white coat syndrome, the use of an inappropriately sized cuff for determining BP or pseudohypertension; (2) insufficient treatment including inadequate adherence to the prescribed medication, insufficient lifestyle modification or the inappropriate use of drugs; (3) the existence of poorly controlled conditions including hypervolemia, obesity, sleep apnea syndrome, excess drinking or the consumption of BP-elevating chemicals or foods and (4) the misdiagnosis of secondary hypertension. It is particularly important to focus on the presence of hypervolemia, which is usually caused by high salt intake in the absence of diuretic treatment and/or the impairment of renal function.

A previous retrospective study recruited patients from two health systems, who had been diagnosed with incidental hypertension from 2002 to 2006, and then selected patients who developed RH or uncontrolled BP despite adherence to $\geqslant 3$ anti-hypertensive medications. ${ }^{9}$ They then examined the associations between medication adherence or treatment intensification and 1-year BP control in order to elucidate which patient characteristics affect $\mathrm{BP}$ control. As a result, they found that $49 \%$ of the $\mathrm{RH}$ patients were male (mean age: 60 years old). They also reported that at 1 year after diagnosis fewer of the $\mathrm{RH}$ patients were taking diuretics, $\beta$-blockers or angiotensinconverting enzyme inhibitors (ACE-I)/angiotensin receptor blockers (ARB) than at the baseline. ${ }^{9}$ Thus, we need to clarify the reason why patients with uncontrolled BP do not undergo treatment intensification. ${ }^{9}$ Moreover, in a study analyzing urine for various anti-hypertensive drugs and their metabolites, it was reported that poor adherence was the most common cause of poor BP control in patients with apparent $\mathrm{RH}$, being twice as common as secondary hypertension. ${ }^{10}$ We therefore should be aware that poorly controlled hypertension under multidrug anti-hypertensive treatment does not always represent true resistance.

\section{ABPM AND ARTERIAL STIFFNESS}

Most cases of hypertension involve a white coat effect, which leads to the overestimation of BP. The prevalence and clinical characteristics of white coat or isolated office $\mathrm{RH}$ are usually determined by comparing clinic BP values with either daytime home BP measurements or awake BP mean values obtained from ABPM. ${ }^{11}$ Thus, so-called RH might include patients with normal or elevated asleep BP mean values. ${ }^{11}$ The authors of the latter study indicated that RH should be classified into isolated-office $\mathrm{RH}$, masked $\mathrm{RH}$ and true $\mathrm{RH}$, and also emphasized that ABPM should be regarded as a clinical requirement for the diagnosis of RH. ${ }^{11}$ Therefore, when assessing patients with suspected $\mathrm{RH}$, we should measure BP using ABPM, rather than with the conventional cuff methods used to determine clinic BP in order to differentiate it from white coat, isolated office and masked hypertension. A previous study investigated the influence of the timing of hypertensive treatment on circadian BP patterns and the degree of $\mathrm{BP}$ control in order to clarify the relevant clinical and laboratory parameters of $\mathrm{RH}$ patients using 48-hour ABPM. ${ }^{12}$ These findings demonstrated that bedtime-based hypertension medication regimens are recommended for the treatment of patients who have been mistakenly judged to be resistant to therapy by conventional cuff methods or after treatment with a morning-based treatment regimen. ${ }^{12}$ It is suggested that we need to consider when patients should be administered anti-hypertensive drugs in order to achieve well-controlled BP levels. Such decisions should be taken on the basis of ABPM measurements of the diurnal changes in BP, which should be taken into account before definitely diagnosing $\mathrm{RH}$.

It was reported that the variations in systolic $\mathrm{BP}$, as estimated by ABPM, were positively correlated with carotid intima-media thickness, pulse wave velocity and the ambulatory arterial stiffness index (AASI), suggesting that ABPM, which is easier to perform at clinics than pulse wave velocity, and assessments of the variability in 24-hour BP might be useful for evaluating arterial stiffness. ${ }^{13}$ On the other hand, it was proposed that home BP variability on a single occasion was correlated with pulse wave velocity, independent of other known 
risk factors, in Japanese patients with type 2 diabetes. ${ }^{14}$ Another study investigated the correlation between circadian variability in BP/AASI and renal function. ${ }^{15}$ They demonstrated that hypertensive patients who displayed greater circadian variability in systolic BP as determined by ABPM exhibited significantly higher resistive index values. In addition, they found that daytime variability in systolic BP was more strongly correlated with resistive index than nighttime BP variability, ${ }^{15}$ suggesting that monitoring both daytime and nighttime BP variability might be useful for assessing pathological conditions in hypertensive patients and preventing cardiovascular disease in $\mathrm{RH}$ patients.

According to The Spanish Ambulatory Blood Pressure Monitoring Registry, 14461 patients fulfilled the criteria for $\mathrm{RH}$, whereas the BP of 13436 hypertensive patients was successfully controlled using three or fewer anti-hypertensive drugs. ${ }^{16}$ The individuals with $\mathrm{RH}$ were older, more obese and had suffered from hypertension for a longer period than the controlled patients. They were also more likely to suffer from diabetes, dyslipidemia, reduced renal function, microalbuminuria or left ventricular hypertrophy and to display a history of cardiovascular events. In multivariate analysis, the duration of hypertension, obesity, abdominal obesity, left ventricular hypertrophy, a reduced estimated glomerular filtration rate and microalbuminuria were found to be independently associated with RH. In addition, ABPM revealed that white coat hypertension is common among $\mathrm{RH}$ patients, as is masked hypertension among patients with apparently controlled BP. ${ }^{16}$ Only the ABPM-based diagnosis of $\mathrm{RH}$ was reported to be an independent predictor of all-cause mortality. ${ }^{17}$ Higher ambulatory BP was reported to be a predictor of cardiovascular morbidity and mortality in RH patients, whereas office BP had no prognostic value. ${ }^{17}$ In another study, the prognostic value of nocturnal BP reduction in $\mathrm{RH}$ was examined in order to evaluate its importance as a predictor of cardiovascular morbidity and mortality. ${ }^{18}$ As a result, it was clearly demonstrated that the nondipping pattern was an independent predictor of the composite end point (hazard ratio, 1.74; 95\% confidence interval, 1.12-2.71) and cardiovascular mortality (hazard ratio, 2.31; 95\% confidence interval, 1.09-4.92) ${ }^{18}$ It was also reported that ABPM provides a more reliable assessment of BP in patients with chronic kidney disease (CKD) and $\mathrm{RH} .{ }^{19}$ Moreover, it is known that $\mathrm{RH}$ and the non-dipping pattern are valuable predictors of cardiovascular outcomes and that several electrocardiographic findings, such as prolonged ventricular repolarization, serial changes in the strain pattern and left ventricular hypertrophy, renal symptoms of albuminuria, and a reduced glomerular filtration rate are powerful cardiovascular risk markers in $\mathrm{RH}^{20}$

\section{CKD AND RH}

The Olivetti Heart Study (OHS), which was performed in southern Italy, aimed to clarify the predictors of $\mathrm{RH}$ in the general population ${ }^{21}$ and found that the incidence of $\mathrm{RH}$ during the 8 -year follow-up period was $10.1 \%$ among hypertensive participants. They concluded that the urinary albumin/creatinine ratio, an elevated fractional excretion of sodium (FENa) value, increased cholesterol levels and a higher basal BP level were independent predictors of $\mathrm{RH} .{ }^{21}$ Thus, it is suggested that microalbuminuria as an indicator of CKD and the degree of sodium retention, as measured by the $\mathrm{FENa}$, might be strong predictors of future $\mathrm{RH}$. It is strongly recommended that dietary sodium restriction be included in hypertension management strategies. ${ }^{22}$ In another study examining the relationship between obstructive sleep apnea (OSA) and RH in CKD and end-stage renal disease, it was reported that the mean systolic BP (SBP) values of the
CKD and end-stage renal disease groups were significantly higher than that of the non-CKD group despite the fact that the former groups were administered more anti-hypertensive medications. ${ }^{23}$ In addition, the $\mathrm{CKD}$ and end-stage renal disease groups exhibited higher incidences of RH and more severe sleep apnea than the nonCKD group. ${ }^{23}$ Thus, we need to check for the presence of sleep apnea in $\mathrm{RH}$.

\section{LIFESTYLE MODIFICATION FOR RH}

It is speculated that unhealthy lifestyle factors might contribute to apparent treatment-RH. ${ }^{24}$ The latter study analyzed lifestyle factors, including obesity, physical inactivity, current smoking, heavy alcohol consumption, a low Dietary Approaches to Stop Hypertension (DASH) diet score and high sodium-to-potassium $(\mathrm{Na} / \mathrm{K})$ intake, in patients with treatment-RH and concluded that unhealthy lifestyle factors were not independently associated with apparent treatment$\mathrm{RH}^{24}$ On the other hand, obese patients often display treatmentresistant arterial hypertension, possibly due to neurohumoral activation. ${ }^{25}$ Moreover, it remains unclear how weight loss strategies and anti-hypertensive treatments should be combined to achieve optimal clinical outcomes. ${ }^{25}$ It is also known that visceral obesity increases the risk of arterial hypertension. ${ }^{26}$ The latter study analyzed 5065 hypertensive patients with visceral obesity and found that, after excluding undertreated patients, $13.9 \%$ had $\mathrm{RH}$. Moreover, $\mathrm{RH}$ was more common in obese individuals with body mass indices of $\geqslant 35$ and morbidly obese individuals. ${ }^{26}$ These findings suggest that under treatment, the underuse of diuretics in multidrug regimens, and the white coat effect are the most common reasons for the overdiagnosis of $\mathrm{RH}$ in patients with visceral obesity and that obesity is an independent risk factor for $\mathrm{RH}^{26}$ It was also reported that higher aortic stiffness is associated with reduced pulse pressure amplification with increasing adiposity in hypertensive African-American adolescents. $^{27}$ Thus, we should pay attention to the existence of obesity when diagnosing $\mathrm{RH}$.

The effect of physical exercise on hypertension, especially $\mathrm{RH}$, is disputed. In a previous study, 50 subjects with $\mathrm{RH}$ were randomly assigned to participate or not participate in an 8-12-week treadmill exercise program, in which the target lactate level was $2.0 \pm 0.5 \mathrm{mmoll}^{-1} .^{28}$ As a result, it was found that physical exercise decreases BP, even in subjects who exhibit poor responses to medical treatment. Thus, physical exercise as well as measures aimed at reducing patients' body weights should be included in therapeutic programs for $\mathrm{RH}^{28}$

\section{MINERALOCORTICOID RECEPTOR ANTAGONIST TREATMENT FOR RH}

Hypertension is a major risk factor for CKD, but although mineralocorticoid receptor antagonists (MRA) have been demonstrated to be effective at managing $\mathrm{RH}$, they are not always used to treat $\mathrm{CKD}$ patients because they induce hyperkalemia. A retrospective study reported that 32 patients with $\mathrm{RH}$ associated with stage $3 \mathrm{CKD}$ were treated with spironolactone and 4 were treated with eplerenone during a median follow-up period of 312 days. ${ }^{29}$ The MRA induced significant decreases in the patients' SBP and diastolic BP, increased their serum potassium levels from $4.0 \pm 0.5$ to $4.4 \pm 0.5 \mathrm{mEq} \mathrm{I}^{-1}$ $(\mathrm{P}=0.0001)$, with the highest value being $5.8 \mathrm{mEq}^{-1}$ and decreased their estimated glomerular filtration rates. ${ }^{29}$ MRA are quite effective at reducing $\mathrm{BP}$ in subjects with $\mathrm{RH}$ associated with stage $3 \mathrm{CKD}$, although close biochemical monitoring is recommended because of the increased risk of hyperkalemia and worsening renal function. ${ }^{29}$ 
It was previously reported that aldosterone levels are correlated with the severity of OSA in $\mathrm{RH}^{30}$, and that the blockade of aldosterone reduces the severity of OSA. Overall, these findings are consistent with excess aldosterone levels contributing to the worsening of underlying OSA. It was suggested that an excess of aldosterone worsens OSA by inducing fluid accumulation within the neck, which can cause upper airway resistance. ${ }^{30}$ They also reported that marked anti-hypertensive effects are observed when MRA are added to the treatment regimens of patients with $\mathrm{RH}$, further suggesting that excess aldosterone levels are an important cause of RH. Furthermore, it has been suggested that a close association exists between hyperaldosteronism, RH and OSA. ${ }^{31}$

In a previous study, compared with the control group, the $\mathrm{RH}$ patients displayed significantly higher aldosterone-to-renin ratios and plasma aldosterone, 24-hour urinary aldosterone (UAldo), brain-type natriuretic peptide and atrial natriuretic peptide levels as well as significantly lower plasma renin activity and serum potassium levels. ${ }^{32}$ The detection of a significant correlation between 24-hour UAldo levels and cortisol excretion suggested that a common stimulus, such as corticotropin, might underlie the excess aldosterone levels observed in patients with RH. ${ }^{32}$ Thus, we need to consider the involvement of excess aldosterone and cortisol levels in the pathogenesis of $\mathrm{RH}$. Excess aldosterone is also considered to have detrimental metabolic effects that contribute to metabolic syndrome and endothelial dysfunction, which in turn contribute to the development of $\mathrm{RH}$ as well as cardiovascular disease and $\mathrm{CKD} .{ }^{33}$ Moreover, elevated aldosterone levels could have a crucial role in promoting insulin resistance and $\mathrm{RH} .{ }^{34}$ Aldosterone enhances the tissue generation of oxygen-free radicals and systemic inflammation, resulting in impaired insulin metabolic signaling, reduced endothelial-mediated vasorelaxation, and associated cardiovascular and renal structural and functional abnormalities. ${ }^{34}$ It was also reported that the cardiovascular and renal abnormalities associated with insulin resistance are mediated, in part, by aldosterone-based non-genomic and genomic signaling through the mineralocorticoid receptor (MR). ${ }^{34}$ Furthermore, primary aldosteronism (PA), a clinical condition in which aldosterone is produced in excess and renin production is suppressed, is common in patients with $\mathrm{RH}$, and recent studies have suggested that excess aldosterone might have a pathophysiological role in the relationship between hypertension and OSA. Obesity is common in patients that display OSA, excess aldosterone production and/or RH, and might link these conditions. ${ }^{35}$ On the other hand, the National Health and Nutrition Examination Survey (NHANES), which was conducted from 2005 to 2008, examined self-reported and study-measured health and sleep characteristics to test the associations between selfreported sleep symptoms and severe/RH in a nationally representative sample of adults. ${ }^{36}$ As a result, it was found that there was no significant association between self-reported snoring/snorting and $\mathrm{RH},{ }^{36}$ suggesting that sleep disturbance is not correlated with $\mathrm{RH}$.

In a previous study, we found that $61(6 \%)$ of 1020 hypertensive patients were diagnosed with PA from 1995 until 1999. ${ }^{37,38}$ In addition, only $18 \%$ of the patients exhibited serum $\mathrm{K}$ levels of $\leqslant 3.3 \mathrm{mEq} \mathrm{l}^{-1}$. Thus, many clinicians seem to misdiagnose PA as essential hypertension because of the absence of hypokalemia. ${ }^{37,38}$ In a subsequent study, we reported that the incidence of PA is unexpectedly high among patients with hypertension and that aldosterone-producing adenoma, which seems to be a main cause of PA, can be treated with unilateral adrenalectomy. ${ }^{39}$ Among the 61 patients with definitely diagnosed PA in the above-mentioned study, 39\% exhibited BP values of $>180 / 110 \mathrm{~mm} \mathrm{Hg}$ at outpatient clinics, ${ }^{37,38}$ although their BP was not analyzed by ABPM. It was also reported that PA displays an incidence of $17-23 \%$ among patients with $\mathrm{RH},{ }^{40}$ suggesting that we should consider the possibility of PA and screen patients with $\mathrm{RH}$ for it in order to protect them from fatal cardiovascular events. Screening for PA is strongly recommended for patients with $\mathrm{RH} .{ }^{41}$ Moreover, a study examining the prevalence of PA among RH patients reported that PA displayed a markedly higher prevalence among subjects with $\mathrm{RH}$ or stage III hypertension than among the general hypertensive population. ${ }^{42}$ Another report investigating the prevalence of PA among $\mathrm{RH}$ patients demonstrated that it was substantially lower than previously reported, although the $\mathrm{RH}$ patients did display a high prevalence of PA-based hyperaldosteronism. ${ }^{43}$

We prospectively examined the prevalence of endocrine hypertension among 1435 untreated hypertensive patients who visited Yokohama Rosai Hospital, Yokohama, and Social Insurance Central General Hospital, Tokyo. The prevalences of PA, Cushing's syndrome including subclinical Cushing's syndrome and Cushing's disease, pheochromocytoma and renovascular hypertension were 7.2, 1.9, 0.3 and $0.5 \%$, respectively. Unilateral adrenalectomy was performed in the 75 patients with unilateral hyperaldosteronism among the 104 patients with PA, 6 patients with Cushing's syndrome, 5 patients with subclinical Cushing's syndrome and 6 patients with pheochromocytoma. Five patients with Cushing's disease were treated with transsphenoidal adenomectomy, and seven patients with renovascular hypertension were treated with percutaneous transluminal angioplasty. One year after the surgery/percutaneous transluminal angioplasty, the hypertension of 74 patients (71\%) had been completely cured, and that of 30 patients (29\%) had been improved under treatment with low doses of anti-hypertensive agents. The prevalence of endocrine hypertension, such as adrenal and renovascular hypertension, was $10.0 \%$ among the 1435 untreated hypertensive patients, and the hypertension cure rate was $71 \%$ among the 104 patients who underwent surgery or percutaneous transluminal angioplasty. These findings clearly demonstrated a high prevalence rate of endocrine hypertension among hypertensive patients, suggesting that we should appropriately screen hypertensive patients in order to differentiate endocrine hypertension from essential hypertension. Secondary endocrine hypertension is strongly associated with $\mathrm{RH}$ and induces cardiovascular events. We should avoid misdiagnosing secondary endocrine hypertension to prevent $\mathrm{RH}$.

Spironolactone has been demonstrated to have an anti-hypertensive effect in patients with ABPM-diagnosed $\mathrm{RH}^{4}{ }^{4}$ In the latter study, 175 patients were subjected to clinical and complementary examinations at the baseline, before being given spironolactone at doses of 25-100 mg per day. As a result, mean reductions of 16 and $9 \mathrm{~mm} \mathrm{Hg}$, respectively, were observed in the patients' 24-hour SBP and diastolic BP (DBP). Their office SBP and DBP also decreased (by 14 and $7 \mathrm{~mm} \mathrm{Hg}$, respectively). ${ }^{44}$ Accordingly, the authors concluded that the administration of spironolactone to patients with true $\mathrm{RH}$ is safe and effective at decreasing their BP, especially in patients with abdominal obesity and lower arterial stiffness. ${ }^{44}$ Spironolactone is also used for cases of PA and RH that do not involve secondary etiologies. It was reported that the dose ranges for these two conditions overlap significantly. ${ }^{45}$ Thus, we cannot differentiate PA from essential $\mathrm{RH}$ according to the BP-lowering effect of spironolactone.

It was also reported that the pathogenesis of MR-associated hypertension in patients with normal plasma aldosterone levels is mediated by MR activation via pathways other than high aldosterone levels, such as increased MR levels, increased MR sensitivity and MR overstimulation by factors such as Racl. ${ }^{46}$ For RH patients with high plasma aldosterone levels, MRA should be given as a first-line therapy, 
whereas for those with normal aldosterone levels, ARB or ACE-I should be given as a first-line therapy, and MRA can be given as an additional treatment. ${ }^{46}$ In Japan, both the selective MRA eplerenone and the non-selective MR antagonist spironolactone are generally used for the treatment of hypertension. ${ }^{47}$ Sato suggested that the roles of spironolactone and eplerenone in the treatment of hypertension should be carefully differentiated, and we agree with this point. ${ }^{47}$

In a study investigating the role of cortisol in the pathogenesis of $\mathrm{RH}$, patients with $\mathrm{RH}$ were screened for the presence of subclinical hypercortisolism using the $1 \mathrm{mg}$ dexamethasone overnight suppression test, and hypercortisolism was confirmed when two saliva samples collected at $2300 \mathrm{~h}$ displayed cortisol levels of at least $3.6 \mathrm{nmoll}^{-1}$. One hundred and twelve patients (prevalence: $26.5 \%$ ) tested positive for hypercortisolism without overt Cushing's syndrome. The patients who produced positive screening results were older, more likely to be males and displayed higher prevalence of diabetes and target organ damage and higher nighttime SBP values than the patients who produced negative screening results. ${ }^{48}$ As a result, the authors concluded that patients with $\mathrm{RH}$ display a relatively high prevalence of subclinical hypercortisolism, which is associated with several markers of worse cardiovascular prognosis. ${ }^{48}$ In addition, increased circulating levels of glucocorticoids are observed in patients with lifestyle-related disorders, which can also activate MR signaling in cardiovascular, adipose, skeletal muscle, neuronal and liver tissue. ${ }^{34}$

\section{RENAL ARTERY DENERVATION AS A TREATMENT FOR RH}

Treatments for RH involving carotid baroreceptor activation or renal sympathetic denervation have recently been developed. On the other hand, it was reported that while these techniques resulted in marked falls in office BP, the associated ambulatory BP reductions were rather modest. The disparity between the office and ambulatory BP reductions might be explained by sympathetic overactivity. ${ }^{49}$ Renal artery denervation $^{50,51}$ was also reported to reduce SBP and DBP in patients with drug-RH and to reduce atrial fibrillation recurrence when combined with pulmonary vein isolation. ${ }^{52}$

\section{CONCLUSION}

Clinicians should be aware that $\mathrm{RH}$ patients are at risk of poor outcomes. We do not know the exact prevalence of $\mathrm{RH}$ among patients with treated hypertension, although it was reported that the prevalence of RH was $\sim 13 \%$ in the Japanese J-HOME study and ranged from $30-50 \%$ in Western countries. The latter figure appears to be too high and might have been affected by the inclusion of patients with uncontrolled hypertension or clinically suspected secondary hypertension. We need to perform further clinical trials for clarifying the exact prevalence of $\mathrm{RH}$, because $\mathrm{Carey}^{53}$ has recently described that the prevalence of $\mathrm{RH}$ is still a matter of debate. Endocrine hypertension is sometimes present among RH patients. We rule out secondary hypertension during hypertensive patients' first visits, that is, before administering any medications, because secondary hypertension including endocrine hypertension can easily evolve into RH. RH patients often exhibit salt retention, which can be caused by excess aldosterone production or increased reninangiotensin system activity due to overactivity of the sympathetic nervous system. We should clarify the pathophysiological mechanisms of the salt retention and sympathetic nervous system activation observed in RH. Furthermore, RH can be treated using aldosterone antagonists or by blockading the renin-angiotensin system, as well as device-based therapy involving renal denervation and carotid baroreceptor activation. We summarize the optimal method for diagnosing and treating $\mathrm{RH}$ in Tables 1 and 2. The speed at which Japan's
Table 1 Diagnosis of resistant hypertension and its complications

\begin{tabular}{ll}
\hline Definition & $\begin{array}{l}\text { Poor control of BP even under treatment with three different } \\
\text { kinds of anti-hypertensive drugs, including diuretics }\end{array}$ \\
Pathogenesis & $\begin{array}{l}\text { 1. White coat syndrome, pseudohypertension } \\
\text { 2. Inadequate adherence to the prescribed medication, } \\
\text { insufficient lifestyle modification, inappropriate usage of drugs } \\
\text { 3. Poorly controlled conditions including hypervolemia } \\
\text { (excess drinking), obesity, sleep apnea syndrome, BP-elevating } \\
\text { chemicals or foods (NSAID, licorice, and so on) } \\
\text { 4. Misdiagnosis of secondary hypertension } \\
\text { Comparing clinic BP values with daytime home BP or the } \\
\text { awake BP mean by ABPM, and confirming true RH, } \\
\text { differentiated from isolated-office RH and masked RH } \\
\text { Diabetes, dyslipidemia, reduced renal function, } \\
\text { microalbuminuria, left ventricular hypertrophy }\end{array}$ \\
Complications
\end{tabular}

Table 2 Treatment of resistant hypertension

\begin{tabular}{ll}
\hline Lifestyles & Weight loss, physical exercise, low sodium diet, \\
& no smoking, reduction of alcohol consumption \\
Medications & Use long-acting combination agents (ACE inhibitor/ARB + \\
& CCB + hydrochlorothiazide) \\
& Add-on mineralocorticoid receptor antagonists \\
& Add-on other anti-hypertensive drug class \\
Device-based therapy & Optional management by renal denervation therapy
\end{tabular}

population, which shows a preference for eating salty foods, is aging is accelerating. Thus, we need to prevent lifestyle-related diseases, including hypertension, especially among aged individuals, as the prevalence of $\mathrm{RH}$-associated complications remains high.

Finally, we need to focus on the existence of $\mathrm{RH}$ among treated hypertensive patients in order to avoid the early onset of fatal cardiovascular events and decrease medical costs.

\section{CONFLICT OF INTEREST}

The authors declare no conflict of interest in association with this study.

\section{ACKNOWLEDGEMENTS}

This study was partly supported by a Grant for Research on Intractable Diseases provided by the Japanese Ministry of Health, Labour and Welfare.

1 Daugherty SL, Powers JD, Magid DJ, Tavel HM, Masoudi FA, Margolis KL, O'Connor PJ, Selby JV, Ho PM. Incidence and prognosis of resistant hypertension in hypertensive patients. Circulation 2012; 125: 1635-1642.

2 Moreno H Jr, Coca A. Resistant and refractory hypertension: Reflection on pathophysiology and terminology. Blood Pressure 2012; 21: 209-210.

3 Cushman WC, Ford CE, Cutler JA, Margolis KL, Davis BR, Grimm RH, Black HR, Hamilton BP, Holland J, Nwachuku C, Papademetriou V, Probstfield J, Wright JT Jr Alderman MH, Weiss RJ, Piller L, Bettencourt J, Walsh SMALLHAT Collaborative Research Group. Success and predictors of blood pressure control in diverse North American settings: The Antihypertensive and Lipid-Lowering Treatment to Prevent Heart Attack Trial (ALLHAT). J Clin Hypertens 2002; 4: 393-404.

4 Black HR, Elliott WJ, Grandits G, Grambsch P, Lucente T, White WB, Neaton JD, Grimm RH Jr, Hansson L, Lacourciere Y, Muller J, Sleight P, Weber MA, Williams G, Wittes J, Zanchetti A, Anders RJfor the CONVINCE Research Group. Principal results of the Controlled Onset Verapamil Investigation of Cardiovascular End Points (CONVINCE) trial. JAMA 2003; 289: 2073-2082.

5 Dahlöf B, Devereux RB, Kjeldsen SE, Julius S, Beevers G, de Faire U, Fyhrquist $F$ Ibsen H, Kristiansson K, Lederballe-Pedersen O, Lindholm LH, Nieminen MS, Omvik P, Oparil S, Wedel HLIFE Study Group. Cardiovascular morbidity and mortality in the Losartan Intervention For Endpoint reduction in hypertension study (LIFE): a randomised trial against atenolol. Lancet 2002; 359: 995-1003. 
6 Brown MJ, Palmer CR, Castaigne A, de Leeuw PW, Mancia G, Rosenthal T, Ruilope LM. Morbidity and mortality in patients randomised to double-blind treatment with a long-acting calcium-channel blocker or diuretic in the International Nifedipine GITS study: Intervention as a Goal in Hypertension Treatment (INSIGHT). Lancet 2000; 356: 366-372.

7 Julius S, Kjeldsen SE, Weber M et al. VALUE trial groupOutcomes in hypertensive patients at high cardiovascular risk treated with regimens based on valsartan or amlodipine: the VALUE randomised trial. Lancet 2004; 363: 2022-2031. RT.

8 Roberie DR, Elliott WJ. What is the prevalence of resistant hypertension in the United States? Curr Opin Cardiol 2012; 27: 386-391.

9 Daugherty SL, Powers JD, Magid DJ, Masoudi FA, Margolis KL, O'Connor PJ, Schmittdiel JA, Ho PM. The association between medication adherence and treatment intensification with blood pressure control in resistant hypertension. Hypertension 2012; 60: 303-309.

10 Jung O, Gechter JL, Wunder C, Paulke A, Bartel C, Geiger H, Toennes SW. Resistant hypertension? Assessment of adherence by toxicological urine analysis. J Hypertens 2013; 31: 766-774.

11 Ríos MT, Domínguez-Sardiña M, Ayala DE, Gomara S, Sineiro E, Pousa L, Callejas PA, Fontao MJ, Fernández JR, Hermida RC. Prevalence and clinical characteristics of isolated-office and true resistant hypertension determined by ambulatory blood pressure monitoring. Chronobiol Int 2013; 30: 207-220.

12 Hermida RC, Ríos MT, Crespo JJ, Moyá A, Domínguez-Sardiña M, Otero A, Sánchez JJ, Mojón A, Fernández JR, Ayala DE. Treatment-time regimen of hypertension medications significantly affects ambulatory blood pressure and clinical characteristics of patients with resistant hypertension. Chronobiol Int 2013; 30: 192-206.

13 García-García A, García-Ortiz L, Recio-Rodríguez JI, Patino-Alonso MC, Agudo-Conde C Rodriguez-Sanchez E, Gómez-Marcos MA. Relationship of 24-h blood pressure variability with vascular structure and function in hypertensive patients. Blood Press Monit 2013; 18: 101-106.

14 Fukui M, Ushigome E, Tanaka M, Hamaguchi M, Tanaka T, Atsuta H, Ohnishi M, Oda Y, Hasegawa G, Nakamura N. Home blood pressure variability on one occasion is a novel factor associated with arterial stiffness in patients with type 2 diabetes. Hypertens Res 2013; 36: 219-225.

15 Kawai T, Ohishi M, Kamide K, Nakama C, Onishi M, Ito N, Takami Y, Takeya Y, Rakugi $\mathrm{H}$. Differences between daytime and nighttime blood pressure variability regarding systemic atherosclerotic change and renal function. Hypertens Res 2013; 36: 232-239.

16 de la Sierra A, Banegas JR, Oliveras A, Gorostidi M, Segura J, de la Cruz JJ, Armario P, Ruilope LM. Clinical differences between resistant hypertensives and patients treated and controlled with three or less drugs. J Hypertens 2012; 30: 1211-1216.

17 Salles GF, Cardoso CR, Muxfeldt ES. Prognostic influence of office and ambulatory blood pressures in resistant hypertension. Arch Intern Med 2008; 168: 2340-2346.

18 Muxfeldt ES, Cardoso CR, Salles GF. Prognostic value of nocturnal blood pressure reduction in resistant hypertension. Arch Intern Med 2009; 169: 874-880.

19 Shafi S, Sarac E, Tran H. Ambulatory blood pressure monitoring in patients with chronic kidney disease and resistant hypertension. J Clin Hypertens 2012; 14: 611-617.

20 de Souza F, Muxfeldt ES, Salles GF. Prognostic factors in resistant hypertension: implications for cardiovascular risk stratification and therapeutic management. Expert Rev Cardiovasc Ther 2012; 10: 735-745.

21 Barbato A, Galletti F, lacone R, Cappuccio FP, Rossi G, Ippolito R, Venezia A, Farinaro E, Strazzullo P. Predictors of resistant hypertension in an unselected sample of an adult male population in Italy. Intern Emerg Med 2012; 7: 343-351.

22 Viera AJ. Resistant hypertension. J Am Board Fam Med 2012; 25: 487-495.

23 Abdel-Kader K, Dohar S, Shah N, Jhamb M, Reis SE, Strollo P, Buysse D, Unruh ML. Resistant hypertension and obstructive sleep apnea in the setting of kidney disease. $J$ Hypertens 2012; 30: 960-966.

24 Shimbo D, Levitan EB, Booth JN 3rd Calhoun DA, Judd SE, Lackland DT, Safford MM, Oparil S, Muntner P. The contributions of unhealthy lifestyle factors to apparent resistant hypertension: findings from the Reasons for Geographic And Racial Differences in Stroke (REGARDS) study. J Hypertens 2013; 31: 370-376.

25 Jordan J, Yumuk V, Schlaich M, Nilsson PM, Zahorska-Markiewicz B, Grassi G, Schmieder RE, Engeli S, Finer N. Joint statement of the European Association for the Study of Obesity and the European Society of Hypertension: obesity and difficult to treat arterial hypertension. J Hypertens 2012; 30: 1047-1055.

26 Holecki M, Duława J, Chudek J. Resistant hypertension in visceral obesity. Eur J Intern Med 2012; 23: 643-648.

27 Pierce GL, Zhu H, Darracott K, Edet I, Bhagatwala J, Huang Y, Dong Y. Arterial stiffness and pulse-pressure amplification in overweight/obese African-American adolescents: relation with higher systolic and pulse pressure. Am J Hypertens 2013; 26: 20-26.

28 Dimeo F, Pagonas N, Seibert F, Arndt R, Zidek W, Westhoff TH. Aerobic exercise reduces blood pressure in resistant hypertension. Hypertension 2012; 60: 653-658.
29 Pisoni R, Acelajado MC, Cartmill FR, Dudenbostel T, Dell'Italia LJ, Cofield SS, Oparil S, Calhoun DA. Long-term effects of aldosterone blockade in resistant hypertension associated with chronic kidney disease. J Hum Hypertens 2012; 26: 502-506.

30 Dudenbostel T, Calhoun DA. Resistant hypertension, obstructive sleep apnoea and aldosterone. J Hum Hypertens 2012; 26: 281-287.

31 Clark D 3rd, Ahmed MI, Calhoun DA. Resistant hypertension and aldosterone: an update. Can J Cardiol 2012; 28: 318-325.

32 Gaddam KK, Nishizaka MK, Pratt-Ubunama MN, Pimenta E, Aban I, Oparil S, Calhoun DA. Characterization of resistant hypertension: association between resistant hypertension, aldosterone, and persistent intravascular volume expansion. Arch Intern Med 2008; 168: 1159-1164.

33 Sowers JR, Whaley-Connell A, Epstein M. Narrative review: the emerging clinical implications of the role of aldosterone in the metabolic syndrome and resistant hypertension. Ann Intern Med 2009; 150: 776-783.

34 Whaley-Connell Adam, Megan S, Johnson S, James R. Special articles aldosterone: role in the cardiometabolic syndrome and resistant hypertension. Prog Cardiovasc Dis 2010; 52: 401-409.

35 Pimenta Eduardo, Calhoun David A, Oparil Suzanne. Obstructive sleep apnea symposium. Sleep apnea, aldosterone, and resistant hypertension. Prog Cardiovasc Dis 2009; $51:$ 371-380.

36 Walia H, Strohl K, Koo B, Seicean A, Seicean S. Are sleep symptoms predictors of resistant hypertension in a population-based sample? Findings from the National Health and Nutritional Examination Survey. J Clin Hypertens 2012; 14: 530-536.

37 Nishikawa T, Omura M. Clinical characteristics of primary aldosteronism: its prevalence and comparative studies on various causes of primary aldosteronism in Yokohama Rosai Hospital. Biomed Pharmacother 2000; 54 (Suppl 1): 83-85.

38 Omura M, Saito J, Yamaguchi K, Kakuta Y, Nishikawa T. Prospective study on the prevalence of secondary hypertension among hypertensive patients visiting a general outpatient clinic in Japan. Hypertens Res 2004; 27: 193-202.

39 Nishikawa T, Saito J, Omura M. Review article-prevalence of primary aldosteronism: should we screen for primary aldosteronism before treating hypertensive patients with medication? Endocrine J 2007; 54: 487-495.

40 Funder JW, Carey RM, Fardella C, Gomez-Sanchez CE, Mantero F, Stowasser M, Young WF Jr, Montori VM. Case detection, diagnosis, and treatment of patients with primary aldosteronism; an Endocrine Society Clinical Practice Guideline. J Clin Endocrinol Metab 2008; 93: 3266-3281.

41 Ito Y, Takeda R, Takeda Y. Subclinical primary aldosteronism. Best Pract Res Clin Endocrinol Metab 2012; 26: 485-495.

42 Hannemann A, Bidlingmaier M, Friedrich N, Manolopoulou J, Spyroglou A, Völzke $H_{\text {, }}$ Beuschlein F, Seissler J, Rettig R, Felix SB, Biffar R, Döring A, Meisinger C, Peters A, Wichmann HE, Nauck M, Wallaschofski H, Reincke M. Screening for primary aldosteronism in hypertensive subjects: results from two German epidemiological studies. Eur J Endocrinol 2012; 167: 7-15.

43 Douma Stella, Petidis Konstantinos, Doumas Michael, Papaefthimiou Panagiota, Triantafyllou Areti, Kartali Niki, Papadopoulos Nikolaos, Vogiatzis Konstantinos, Zamboulis Chrysanthos. Prevalence of primary hyperaldosteronism in resistant hypertension: a retrospective observational study. Lancet 2008; 371: 1921-1926.

44 de Souza F, Muxfeldt E, Fiszman R, Salles G. Efficacy of spironolactone therapy in patients with true resistant hypertension. Hypertension 2010; 55: 147-152.

45 Handler J. Overlapping spironolactone dosing in primary aldosteronism and resistant essential hypertension. J Clinic Hypert 2012; 14: 732-734.

46 Shibata $\mathrm{H}$, Itoh $\mathrm{H}$. Mineralocorticoid receptor-associated hypertension and its organ damage: clinical relevance for resistant hypertension. Am J Hypertens 2012; 25: 514-523.

47 Sato A. Mineralocorticoid receptor antagonists: their use and differentiation in Japan. Hypertens Res 2013; 36: 185-190.

48 Martins LC, Conceição FL, Muxfeldt ES, Salles GF. Prevalence and associated factors of subclinical hypercortisolism in patients with resistant hypertension. J Hypertens 2012; 30: 967-973.

49 Doumas M, Anyfanti P, Bakris G. Should ambulatory blood pressure monitoring be mandatory for future studies in resistant hypertension: a perspective. J Hypertens 2012; 30: 874-876.

50 Grassi G, Mancia G. New therapeutic approaches for resistant hypertension. J Nephrol 2012; 25: 276-281.

51 Ahmed H, Neuzil P, Skoda J, Petru J, Sediva L, Schejbalova M, Reddy VY. Renal sympathetic denervation using an irrigated radiofrequency ablation catheter for the management of drug-resistant hypertension. JACC Cardiovasc Interv 2012; 5: 758-765.

52 Pokushalov E, Romanov A, Corbucci G, Artyomenko S, Baranova V, Turov A, Shirokova N, Karaskov A, Mittal S, Steinberg JSA. randomized comparison of pulmonary vein isolation with versus without concomitant renal artery denervation in patients with refractory symptomatic atrial fibrillation and resistant hypertension. J Am Coll Cardiol 2012; 60: $1163-1170$.

53 Carey RM. Resistant hypertension. Hypertension 2013; 61: 746-750. 